EPJ Web of Conferences 66, 01019 (2014)

DOI: 10.1051/epjconf/ 20146601019

(C) Owned by the authors, published by EDP Sciences, 2014

\title{
Shell structure, emerging collectivity, and valence $p-n$ interactions
}

\author{
R.B. Cakirli ${ }^{1,2}$ \\ ${ }^{1}$ Department of Physics, University of Istanbul, Istanbul, Turkey \\ ${ }^{2}$ Max-Planck-Institut für Kernphysik, Saupfercheckweg 1, D-69117 Heidelberg, Germany
}

\begin{abstract}
The structure of atomic nuclei depends on the interactions of its constituents, protons and neutrons. These interactions play a key role in the development of configuration mixing and in the onset of collectivity and deformation, in changes to the single particle energies and magic numbers, and in the microscopic origins of phase transitional behavior. Particularly important are the valence proton-neutron interactions which can be studied experimentally using double differences of binding energies extracted from high-precision mass measurements. The resulting quantities, called $\delta \mathrm{V}_{\mathrm{pn}}$, are average interaction strengths between the last two protons and the last two neutrons. Focusing on the $\mathrm{Z}=50-82, \mathrm{~N}=82-126$ shells, we have considered a number of aspects of these interactions, ranging from their relation to the underlying orbits, their behaviour near close shells and throughout major shells, their relation to the onset of collectivity and deformation, and the appearance of unexpected spikes in $\delta \mathrm{V}_{\mathrm{pn}}$ values for a special set of heavy nuclei with nearly equal numbers of valence protons and neutrons. We have calculated spatial overlaps between proton and neutron Nilsson orbits and compared these with the experimental results. Finally we also address the relation between masses (separation energies), changes in structure and valence nucleon number.
\end{abstract}

\section{INTRODUCTION AND AVERAGE PROTON-NEUTRON INTERACTIONS AND EMERGING COLLECTIVITY}

The numbers of protons and neutrons and/or valence protons and neutrons can guide us in understanding special properties about a nucleus or a nuclear region such as the onset of deformation, shape changes and so on. The importance of the number of valence particles is already embodied in concepts such as the $\mathrm{N}_{\mathrm{p}} \mathrm{N}_{\mathrm{n}}$ scheme and the P-factor [1]. Concerning actual observables, in particular for even-even nuclei, we as nuclear structure physicists often first look at the energy of the first excited $2^{+}$state $\mathrm{E}\left(2_{1}{ }^{+}\right), \mathrm{R}_{4 / 2}$ which is a ratio of the first excited $4^{+}$over the first excited $2^{+}$level energies, $\mathrm{B}\left(\mathrm{E} 2 ; 2^{+} \rightarrow 0^{+}\right)$, charge radii, masses (hence binding and separation energies), etc. To gain an idea about structure, Figure 1 illustrates experimental $\mathrm{E}\left(2_{1}{ }^{+}\right.$) (left) and $\mathrm{R}_{4 / 2}$ (right) values for almost the entire nuclei chart. The colors change from red around magic numbers (magicity) through blue around mid-shell (deformation). High (small) $\mathrm{E}\left(2_{1}{ }^{+}\right)$(the $R_{4 / 2}$ ratio) values are seen at and also close to magic numbers. Lower $\mathrm{E}\left(2_{1}{ }^{+}\right.$) (larger $R_{4 / 2}$ ) occur towards midshell as expected. In both panels in Fig. 1 , this is clearly seen and the similarity in the two panels is spectacular. One of the most important challenges relating to Fig. 1 is whether this remarkable pattern will continue for nuclei far from

This is an Open Access article distributed under the terms of the Creative Commons Attribution License 2.0, which permits unrestricted use, distribution, and reproduction in any medium, provided the original work is properly cited. 
stability. For this, future experiments, in particular in the new facilities such as FAIR, FRIB, RIKEN, are needed.

Masses reflect all the nucleonic interactions and thus play a special role as an observable. However, the mass itself does not easily give information on nuclei directly due to its scale $(\sim 1 \mathrm{GeV}$ per nucleon). Thus, one works with differences of binding energies (or masses), separation energies. One can then easily see, in particular, shell effects at the magic numbers, or the onset of deformation, for example in the $\mathrm{N} \sim 90$ region. In addition to differences of binding energies for separation energies, a specific double difference of binding energies offers a special filter for proton (p) - neutron (n) interactions between the last two protons and the last two neutrons [2-5]. The p-n interaction is important because it helps to understand, for example, magic numbers, deformation, single particle energies, collectivity, and the development of configuration mixing. There are many studies in the literature on the p-n interaction [6]. In this Proceedings, we will only talk about average valence $\mathrm{p}-\mathrm{n}$ interactions, called $\delta \mathrm{V}_{\mathrm{pn}}$, between $\mathrm{Z}^{\text {th }}$ and $(\mathrm{Z}-1)^{\text {th }}$ protons with $\mathrm{N}^{\text {th }}$ and $(\mathrm{N}-1)^{\text {th }}$ neutrons. Equation (1) shows this special filter for $\mathrm{p}$-n interactions using binding energies, $\mathrm{B}$, for even-even nuclei.

$$
\left|\delta \mathrm{V}_{\mathrm{pn}}(\mathrm{Z}, \mathrm{N})\right|=1 / 4[((\mathrm{~B}(\mathrm{Z}, \mathrm{N})-\mathrm{B}(\mathrm{Z}, \mathrm{N}-2))-\mathrm{B}(\mathrm{Z}-2, \mathrm{~N})-\mathrm{B}(\mathrm{Z}-2, \mathrm{~N}-2))]
$$
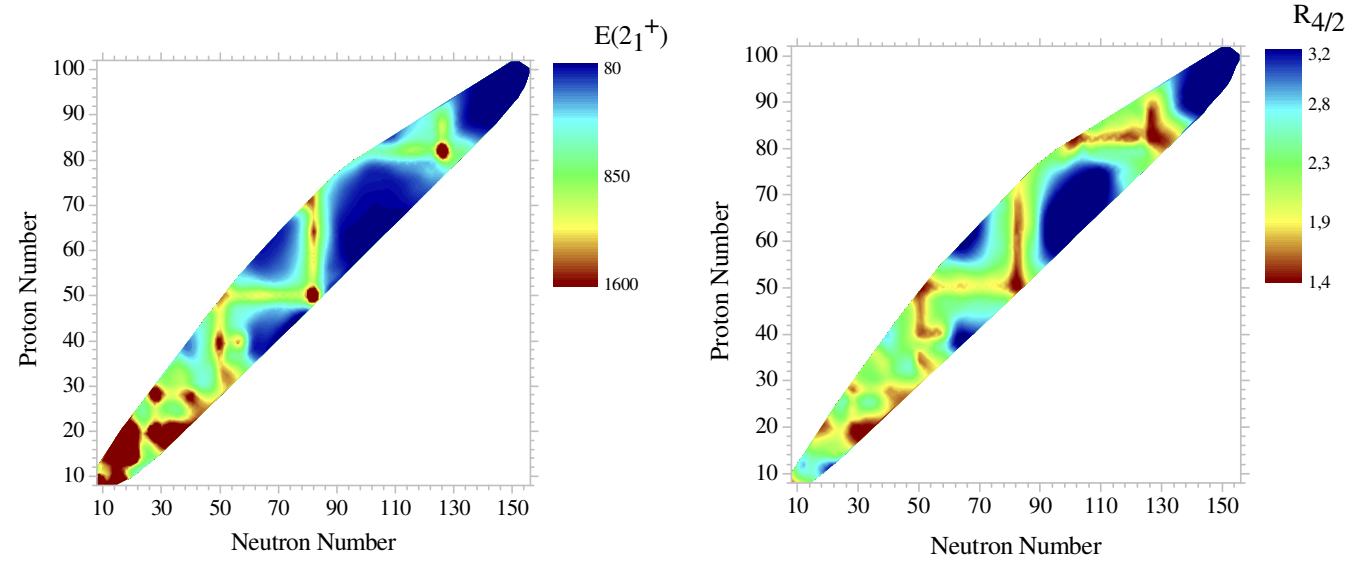

Figure 1. Color coded experimental $\mathrm{E}\left(2_{1}^{+}\right)$(Left) and $\mathrm{R}_{4 / 2}$ (Right) values versus neutron number.

Figure 2 (Left) reveals experimental $\delta \mathrm{V}_{\mathrm{pn}}$ values around the ${ }^{208} \mathrm{~Pb}$ region [3,5]. Since the nuclei presented in Fig. 2 are close to magic numbers, our simple interpretation here is based on Shell Model orbits, $\mathrm{nl}_{\mathrm{j}}$. In the Shell Model, except for the unique parity orbits, each shell begins with high j-low $\mathrm{n}$ and ends with low j-high $\mathrm{n}$ (see Fig. 2 (Right)). If protons and neutrons fill similar orbits $\left(\mathrm{nl}_{\mathrm{j}}\right)$, the expected overlap between $\mathrm{p}$ and $\mathrm{n}$ wave functions will be large. The easiest example is a doubly magic nucleus ${ }^{208} \mathrm{~Pb}$ in which both protons and neutrons are just below the magic numbers $\mathrm{Z}=82$ and $\mathrm{N}=126$, respectively. Thus the last two protons and the last two neutrons occupy similar orbits (low j-high $\mathrm{n}$ ), so we observe a large $\delta \mathrm{V}_{\mathrm{pn}}$ as expected. As soon as two additional neutrons are added to ${ }^{208} \mathrm{~Pb}\left({ }^{210} \mathrm{~Pb}\right)$, these two neutrons will cross the $\mathrm{N}=126$ magic number and fill the beginning of the next shell although protons are still at the end of the 50-82 shell. For this case, the valence protons and neutrons in ${ }^{210} \mathrm{~Pb}$ will have dissimilar $\mathrm{nl}_{\mathrm{j}}$ so we see a sudden drop in $\delta \mathrm{V}_{\mathrm{pn}}$ at $\mathrm{N}=128$ for $\mathrm{Pb}$ [5]. A similar analysis can be done for other nuclei close to magic numbers, but this simple interpretation does not work for deformed nuclei because the orbits occupied by protons and neutrons are complex admixtures of Shell Model configurations. Another facet about the interpretation of $\delta \mathrm{V}_{\mathrm{pn}}$ is that some effects are much clearer in odd-A nuclei compared to even-even nuclei due to pairing effects (see below). 

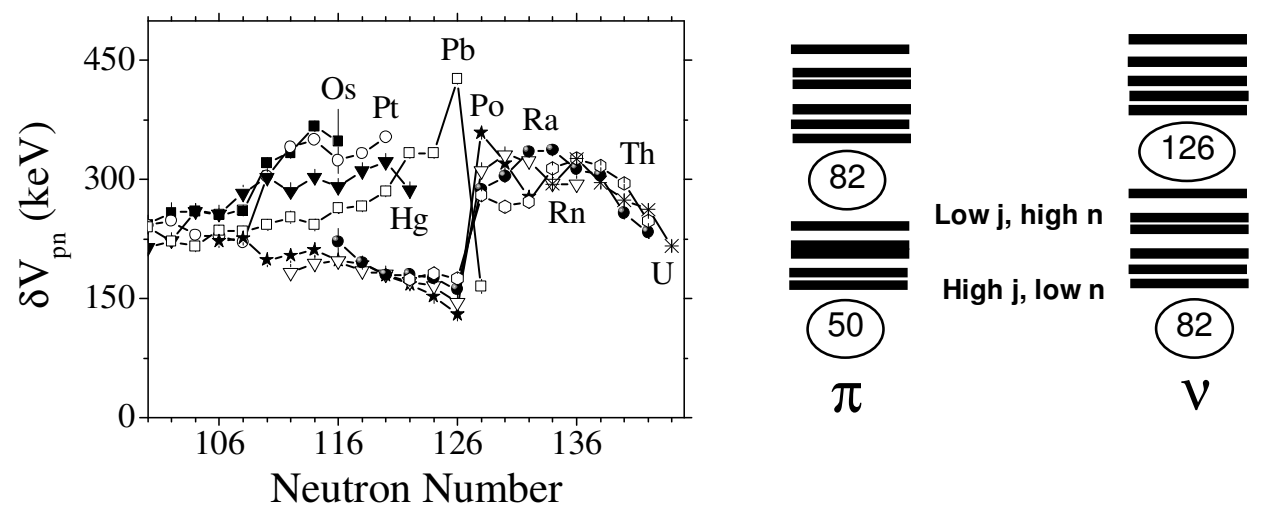

Figure 2. (Left) Experimental $\delta \mathrm{V}_{\mathrm{pn}}$ values versus neutron number for the ${ }^{208} \mathrm{~Pb}$ region. (Right) A toy scheme considering the Shell Model orbits to reveal how $\mathrm{j}$ and $\mathrm{n}$ change within a shell and the expected relation to $\delta \mathrm{V}_{\mathrm{pn}}$ values. Based on Refs. [3,5].

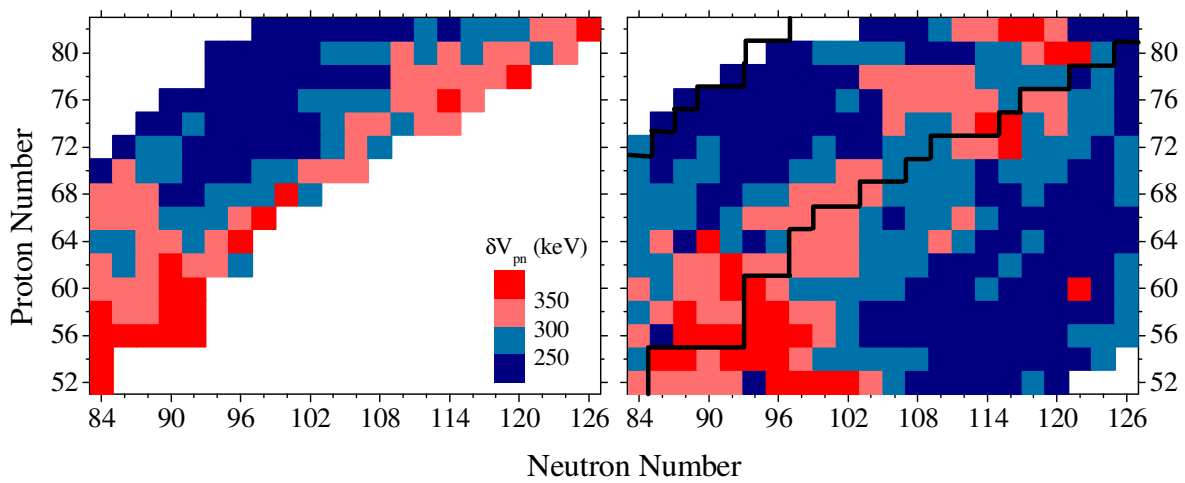

Figure 3. Color coded $\delta \mathrm{V}_{\mathrm{pn}}$ values for both experiment (Left) and DFT (Right). Black zig-zag lines are the boundary of experimentally known $\delta \mathrm{V}_{\mathrm{pn}}$. Based on Ref. [5].

Experimental and calculated $\delta \mathrm{V}_{\mathrm{pn}}$ values, using nuclear density functional theory (DFT), for eveneven nuclei in the $\mathrm{Z}=50-82, \mathrm{~N}=82-126$ shells are presented in Fig. 3. Red and pink colors refer to large $\delta \mathrm{V}_{\mathrm{pn}}$ values and dark and light blue are for low $\delta \mathrm{V}_{\mathrm{pn}}$ values. The DFT results clearly have very good agreement with the experimental results. In general, the largest $\delta \mathrm{V}_{\mathrm{pn}}$ values are in the particle-particle (pp) region in both theory and data and the smallest $\delta \mathrm{V}_{\mathrm{pn}} \mathrm{s}$ are hole-particle (hp). The largest $\delta \mathrm{V}_{\mathrm{pn}} \mathrm{s}$ are located around the diagonal from $\mathrm{Z}=52, \mathrm{~N}=84$ to $\mathrm{Z}=82, \mathrm{~N}=126$. Recent Xe mass measurements [7] at CERN-ISOLTRAP provided the first empirical $\delta \mathrm{V}_{\mathrm{pn}}$ values for $\mathrm{Ba}$ at $\mathrm{N}=90$ and 92 . The DFT results for these recent $\delta \mathrm{V}_{\mathrm{pn}}$ values compare very well with these new data. Calculated $\delta \mathrm{V}_{\mathrm{pn}}$ values are also shown for all the nuclei within the expected driplines) in the $\mathrm{Z}=50-82, \mathrm{~N}=82-126$ shells. Since there is no data in the lower right quadrant (ph), we cannot judge the theory there but clearly this and the right hand side of the (pp) quadrant are prime testing grounds for future exotic beam mass measurements. Thus, some projects are in progress for future mass measurements.

Figures 2 and 3 show heavy nuclei which occupy the large shells (see Fig. 2 (right)) typical of heavy nuclei. In contrast to heavy nuclei, light nuclei fill smaller shells and therefore collective effects appear/disappear quickly so changes in structure occur faster than in heavy nuclei. In addition, light nuclei have cases of nuclei with $\mathrm{Z}=\mathrm{N}$. In terms of $\delta \mathrm{V}_{\mathrm{pn}}$, nuclei with equal numbers of proton and neutron fill the same orbits so maximal spatial-spin overlap of proton and neutron wave functions is expected. Not surprisingly $\mathrm{Z}=\mathrm{N}$ nuclei exhibit large singularities in $\delta \mathrm{V}_{\mathrm{pn}}$ [8] which have been linked [9] to Wigner $\mathrm{SU}(4)$ supermultiplet theory [10]. Figure 4 (Left) shows empirical $\delta \mathrm{V}_{\mathrm{pn}}$ values as a 
function of neutron number for light nuclei with sharp peaks at $\mathrm{Z}=\mathrm{N}$. This clear trend (see the scale) is not expected in heavy nuclei because of the Coulomb force and the spin-orbit force which brings unique parity orbits into each shell. However, for heavy nuclei, we have found that $\delta \mathrm{V}_{\mathrm{pn}}$ values also have sharp peaks, and that they occur when a nucleus has approximately the same number of valence (val) protons and neutrons [11,12]. Figure 4 (Middle) introduces our point for odd-Z with both even and odd-N nuclei (see also Fig. 3 of Ref. [11] where $\delta V_{p n}$ in even-even nuclei also show peaks for $\mathrm{Z}_{\mathrm{val}} \sim \mathrm{N}_{\mathrm{val}}$ but the peaks are suppressed and smoother due to pairing effects). The top axis in the middle panel gives the number of valence neutrons. For example, we see a peak in $\delta \mathrm{V}_{\mathrm{pn}}$ at $\mathrm{N}=95\left(\mathrm{~N}_{\mathrm{val}}=15\right)$ for ${ }_{65} \mathrm{~Tb}\left(\mathrm{Z}_{\mathrm{val}}=15\right)$. Similar examples are seen for Ho and so on. The right panel of Fig. 4 shows the locus of the maximum $\delta \mathrm{V}_{\mathrm{pn}}$ values (white circles) on an Z-N contour plot for $\mathrm{R}_{4 / 2}$ in which structure changes from spherical (blue) through deformed, ellipsoidal shapes (red). The black line is placed to indicate $\mathrm{Z}_{\mathrm{val}}=\mathrm{N}_{\mathrm{val}}$. As seen in this plot, the nuclei for which we have peaks in the experimental $\delta \mathrm{V}_{\mathrm{pn}}$ values, lie where the saturation of collectivity starts so these maxima in $\delta \mathrm{V}_{\mathrm{pn}}$ have a clear link to the evolution of collectivity.
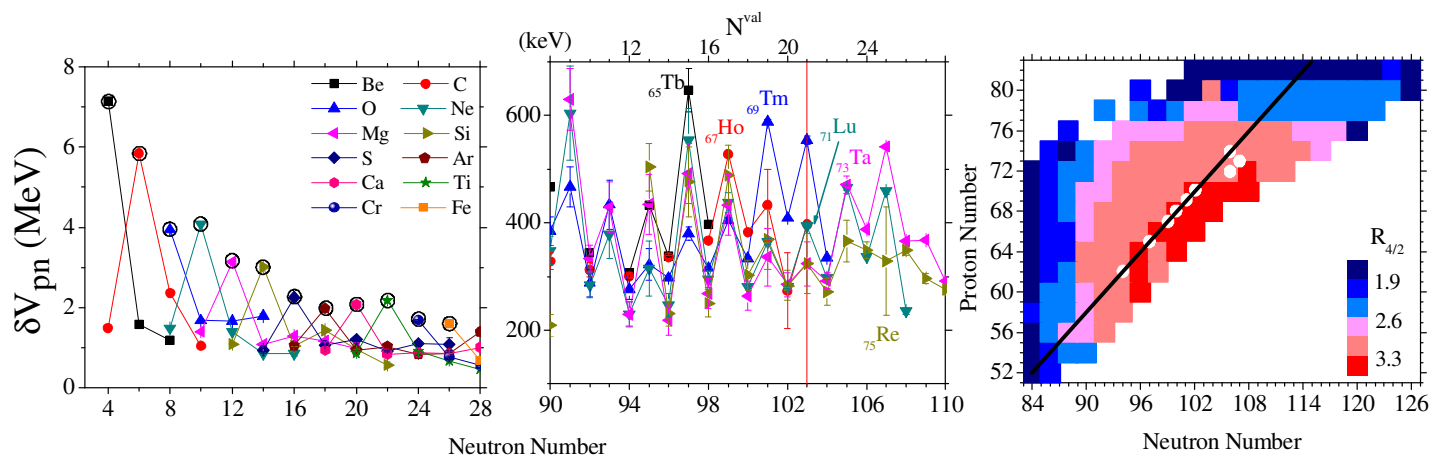

Figure 4. Experimental $\delta \mathrm{V}_{\mathrm{pn}}$ values for light nuclei (Left) and heavy nuclei (Middle). (Right): Color coded $\mathrm{R}_{4 / 2}$ values. White circles are shown for the nuclei for which there is a maximum in $\delta \mathrm{V}_{\mathrm{pn}}$ in the Middle panel. Based on Refs. [12,13].

One of the most interesting points about these results is that these deformed nuclei where we have peaks at $\mathrm{Z}_{\mathrm{val}}=\mathrm{N}_{\mathrm{val}}$ or $\mathrm{Z}_{\mathrm{val}}=\mathrm{N}_{\mathrm{val}} \pm 1$ usually have a very specific relation between the last filled proton and neutron Nilsson orbits, namely that their quantum numbers are related by $\delta \mathrm{K}\left[\delta \mathrm{N}, \delta \mathrm{n}_{\mathrm{z}}, \delta \Lambda\right]$ $=0[110]$ (for even-even nuclei, the best example is ${ }^{168} \mathrm{Er}$ which has $7 / 2[523]\left(\mathrm{K}\left[\mathrm{N}, \mathrm{n}_{\mathrm{z}}, \Lambda\right]\right) \mathrm{p}$ Nilsson orbit and 7/2[633] $n$ Nilsson orbit with $Z_{\mathrm{val}}=\mathrm{N}_{\mathrm{val}}=18$ ). Is this maximum in $\delta \mathrm{V}_{\mathrm{pn}}$ with 0 [110] where the saturation of collectivity starts accidental? In order to understand these interactions in a simple way, we calculated [12] spatial overlaps of proton and neutron Nilsson wave functions with three deformations, namely $0.05,0.22,0.3$. The details of these calculations are given (and updated) elsewhere $[13,14]$. Here we focus on the interpretation and results.

Figure 5 illustrates the Nilsson orbits for protons (Left) in the 50-82 shell and neutrons (Right) in the 82-126 shell. When one starts to fill the proton and neutron Nilsson orbits synchronously, the difference between proton and neutron Nilsson orbits is usually 0[110] such as in the first Nilsson orbits in both shells, 1/2[431] proton orbit with 1/2[541] neutron orbit (follow the same colors in both panels). Because the Nilsson diagrams for the protons and neutrons have similar shapes and crossings, this approximate synchronization actually persists as more nucleons are added and as deformation sets in. Since the proton and neutron shells in Fig. 5 do not have the same size, however, some neutron orbits will not have a proton partner related by 0[110]. These neutron orbits are shown by solid/dotted/dashed gray lines. Interestingly, each of these neutron orbits has $n_{z}=0$ which does not contribute to prolate deformation but to oblate. 


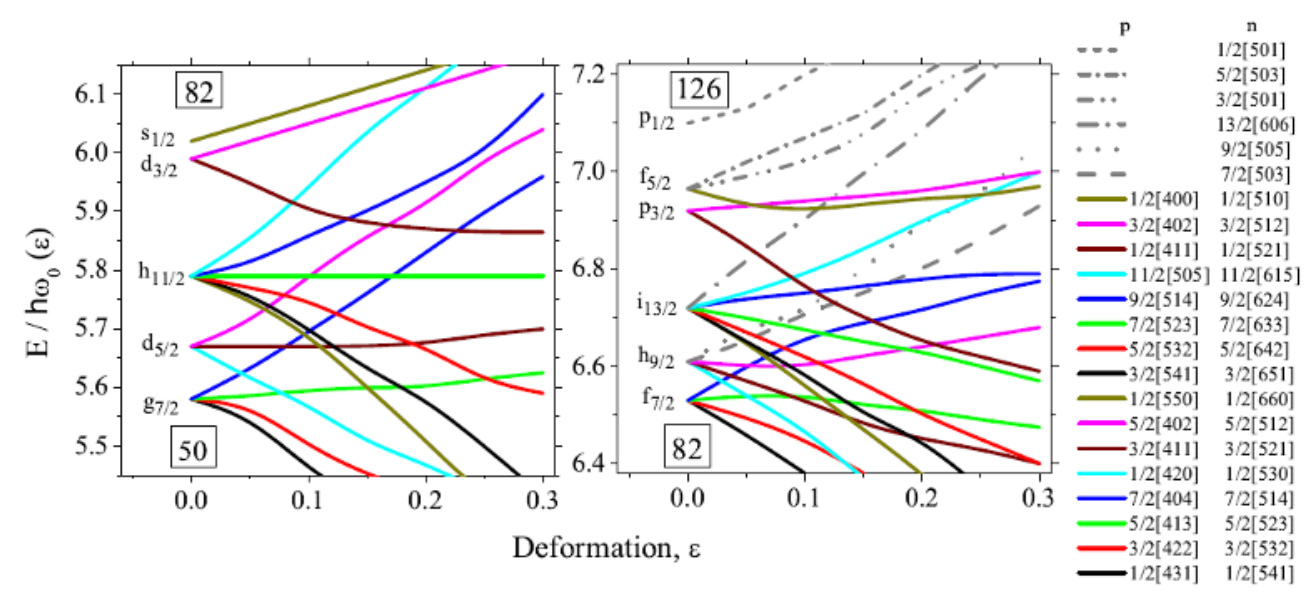

Figure 5. Nilsson diagrams for the proton $\mathrm{Z}=50-82$ and neutron $\mathrm{N}=82-126$ shells (see text for details). Based on Refs. [12,13].

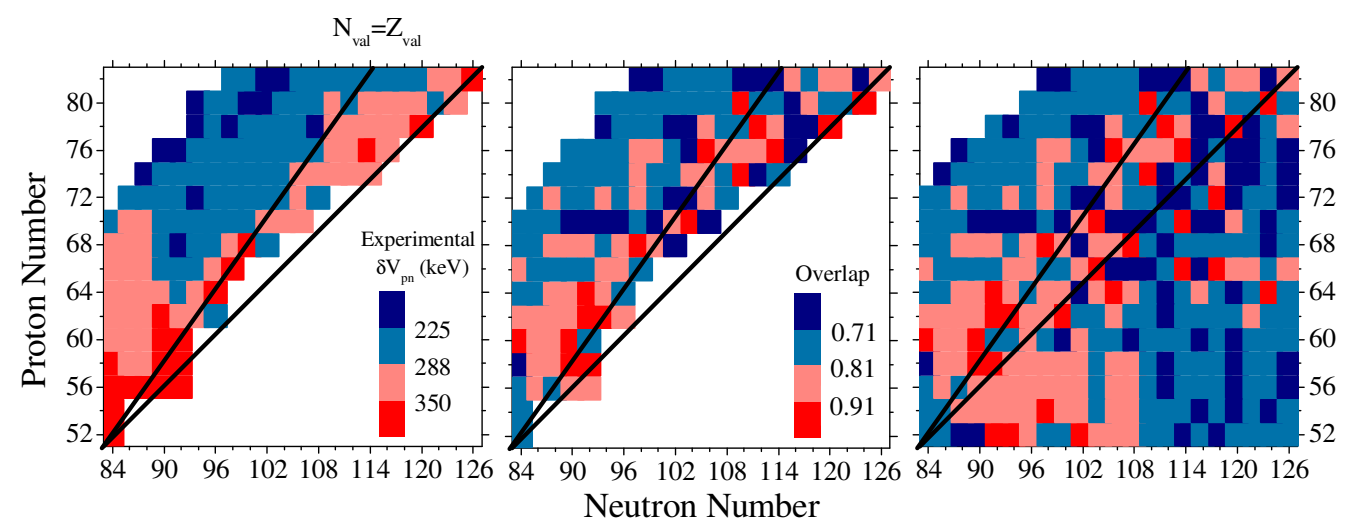

Figure 6. (Left): Color coded empirical $\delta \mathrm{V}_{\mathrm{pn}}$ values for the $\mathrm{Z}=50-82$ and $\mathrm{N}=82-126$ shells. Large values have redder colors. (Middle): Similar as (Left) but for calculated overlaps for nuclei where empirical values of $\delta V_{\text {pn }}$ are known. (Right): Calculated overlaps for the full major shells (excluding the nuclei beyond the proton dripline at far upper left). (see text for details). Based on Ref. [13].

The calculated overlaps are compared with the experimental $\delta \mathrm{V}_{\mathrm{pn}}$ results in Fig. 6. A diagonal line is placed to show where the neutron and proton orbits fill similar fractions of their respective shells. One can fill 32 nucleons into the proton shell and 44 nucleons into the neutron shell. Thus, the figures are designed as square so that, that along the diagonal line similar orbits will be filled so large overlaps (large $\delta \mathrm{V}_{\mathrm{pn}}$ ) are expected along this line (see explanation above $\mathrm{nl}_{\mathrm{j}}$ ) [4]. The upper black line is placed to show the $\mathrm{Z}_{\mathrm{val}}=\mathrm{N}_{\mathrm{val}}$ nuclei. Overall agreement between the data and theory is very good. As mentioned in the DFT results above, the largest $\delta \mathrm{V}_{\mathrm{pn}}$ values are in the pp region and large overlaps along the diagonal lines are seen. There are some discrepancies in the upper right in Fig. 6. However, this is not so surprising because disagreement may happen due to a $\gamma$-soft structure that our calculations do not take into account [13]. In addition, we observe large $\delta \mathrm{V}_{\mathrm{pn}}$ values with $0[101]$ relation (not 0[110]) around $\mathrm{Z}$ 52-64 with $\mathrm{N} \sim 92-108(5 / 2[413] \mathrm{p}-5 / 2[512] \mathrm{n}$ and 1/2[420] $\mathrm{p}$ $1 / 2[521] \mathrm{n}$ orbits). Mass measurements in this region will help us to understand the $\mathrm{p}$ - $\mathrm{n}$ interactions more. 


\section{RELATION BETWEEN $S_{2 \mathrm{~N}}$ AND $\mathrm{E}\left(2_{1}{ }^{+}\right)$VALUES USING RECENT SCHOTTKY MASS MEASUREMENTS}

Masses of ${ }^{181,183} \mathrm{Lu},{ }^{185,186} \mathrm{Hf},{ }^{187,188} \mathrm{Ta},{ }^{191} \mathrm{~W}$, and ${ }^{192,193} \mathrm{Re}$ nuclei were measured [14] for the first time using Storage-ring mass spectrometry with neutron-rich ${ }^{197} \mathrm{Au}$ projectile fragments. With the new masses, two-neutron separation energies, $S_{2 n}$, were investigated. Figure 7 (Left) shows a region where the first $S_{2 n}$ values using the new masses at $\mathrm{Hf}$ with $\mathrm{N}=113,114$ and $\mathrm{W}$ with $\mathrm{N}=117$ are covered with a circle/elipsoid and where the open symbols for $\mathrm{S}_{2 \mathrm{n}}$ in $\mathrm{W}$ at $\mathrm{N}=115$ and 116 and $\mathrm{Os}$ at $\mathrm{N}=119$ are from the previous literature. In Ref. [14], we improved the previous results. Figure 7 (Right) shows experimental $\mathrm{E}\left(2_{1}{ }^{+}\right)$values as a function of neutron number. Using these two useful observables, we have identified a link between observed irregularities in the smooth two-neutron separation energies for $\mathrm{Hf}$ and $\mathrm{W}$ isotopes and changes in collectivity in the corresponding nuclei [14].
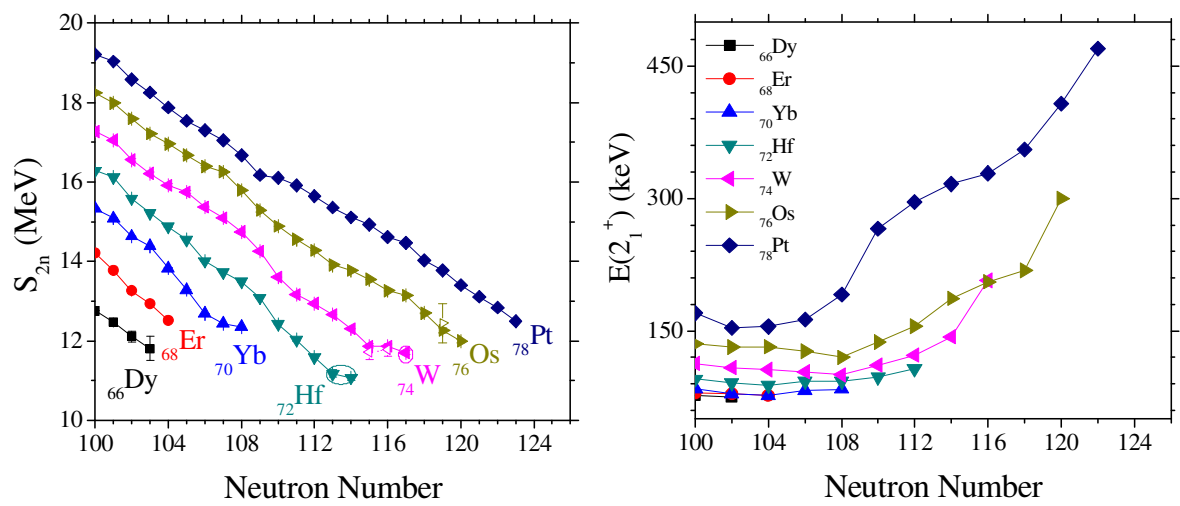

Figure 7. Experimental two neutron separation energies (Left) and the first excited $2^{+}$energies (Right) as a function of neutron number. Based on Ref. [14].

There is a clear flattening in $\mathrm{S}_{2 \mathrm{n}}$ for $\mathrm{W}$ which starts at $\mathrm{N}=116$ and $\mathrm{E}\left(2_{1}^{+}\right)$at $\mathrm{N}=116$ has a jump. For $\mathrm{Pt}$, from $\mathrm{N}=108$ to 110 , we see a similar result as in $\mathrm{W}$. It looks like there is a turning point as a structural change. However, in Os from $\mathrm{N}=118$ to 120 , there is a smooth decrease in $\mathrm{S}_{2 \mathrm{n}}$ although there is a visible increase in $\mathrm{E}\left(2_{1}{ }^{+}\right)$. The Pt isotopes around $\mathrm{N}=120$ are the same. Our interpretation here is based on the number of valence nucleons for these nuclei. Figure 8 is helpful to explain our interpretation. It shows a symmetry triangle with a color code in binding energy from the IBA-1 [15]. The three corners show three dynamical symmetries and the triangles are presented for boson numbers of 5 and 16 (valence nucleon numbers 10 and 32). The color code scale is the same for both triangles and the triangles show changes from left to right from small binding energy (yellow) to large (red). What is seen in Fig. 8 is that when we have larger boson numbers, larger binding comes into the triangle. That is, binding energy increases rapidly with valence nucleon number because binding energy depends roughly on the number of valence nucleons squared. In addition, the binding decreases from deformed (axially deformed, SU(3)) to vibrational (U(5)).

Considering this valence nucleon effect on binding, the nuclei in Fig. 7 mentioned above can be explained. $\mathrm{W}$ at $\mathrm{N}=116$ and $\mathrm{Pt}$ at $\mathrm{N}=110$ have 18 and 20 valence nucleons, respectively. However, Os and $\mathrm{Pt}$ at $\mathrm{N}=120$ have 12 and 10 valence nucleons. Also, we go from SU(3) corner through middle of the triangle in these latter nuclei, so the scale of binding is smaller to begin with. This change means that $S_{2 n}$ values will be smaller. Hence, one does not see a visible change in $S_{2 n}$ for Os and (the heavy) Pt isotopes in Fig. 7 although there is an effect for $\mathrm{W}$ and the lighter Pt isotopes. Taking into account both the dependence of binding on structure (position within the triangle) and the scale of binding as a function of valence nucleon number gives a very useful way to make an easy correlation between $S_{2 n}$ and $\mathrm{E}\left(2_{1}{ }^{+}\right)$. As a result, well deformed nuclei (many bosons) show observable changes in $\mathrm{S}_{2 \mathrm{n}}$ if there is a visible change in $\mathrm{E}\left(2_{1}^{+}\right)$. For more details, see Ref. [14]. 


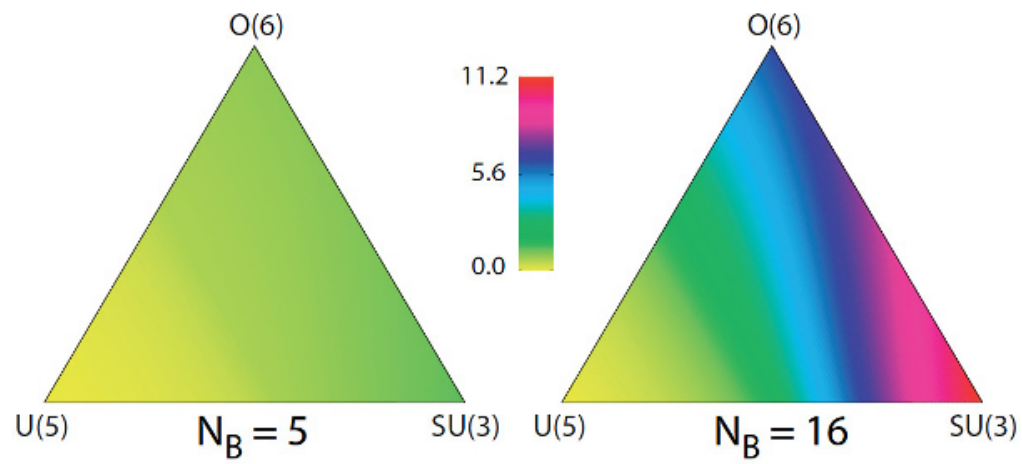

Figure 8. The symmetry triangle of the IBA showing the three dynamical symmetries at the vertices. The colors indicate calculated collective contributions in $\mathrm{MeV}$ to binding energies for $\mathrm{N}_{\mathrm{B}}=5$ (Left) and $\mathrm{N}_{\mathrm{B}}=16$ (Right). Based on Refs. [14, 16].

\section{CONCLUSION}

The average valence proton-neutron interactions, $\delta \mathrm{V}_{\mathrm{pn}}$, which can be studied experimentally using double differences of binding energies extracted from high-precision mass measurements, are discussed from both experimental and theoretical perspectives by focusing on the $\mathrm{Z}=50-82, \mathrm{~N}=82-126$ shells. We noted the correlation between large $\delta \mathrm{V}_{\mathrm{pn}}$ values and the growth of collectivity and the unique behaviour of $\delta \mathrm{V}_{\mathrm{pn}}$ when crossing magic numbers. Reflecting the clear spikes in $\delta \mathrm{V}_{\mathrm{pn}}$ for light nuclei, studies of $\delta \mathrm{V}_{\mathrm{pn}}$ for heavy nuclei are discussed in terms of the number of valence nucleons. We obtained a systematic behavior (maxima in $\delta \mathrm{V}_{\mathrm{pn}}$ ) when a nucleus has equal (or almost equal) numbers of valence protons and neutrons and noticed a consistent difference between proton and neutron Nilsson orbit quantum numbers, namely 0[110]. Calculated spatial overlaps between proton and neutron Nilsson orbits are presented and compared these with the experimental results. Finally, we also discussed the relation between masses (separation energies) and $\mathrm{E}\left(2_{1}{ }^{+}\right)$by taking into account the number of valence nucleons.

\section{ACKNOWLEDGMENTS}

We are grateful to R. F. Casten, K. Blaum, D. Bonatsos, Y. Litvinov and D. Shubina for very useful discussions. This work was supported by the Humboldt Fellowship for R.B.C., by the Max-Planck Society and by the Istanbul University Scientific Research Projects, Numbers. 33027, 26433 and 21658.

\section{References}

1. R.F. Casten, D.S. Brenner and P.E. Haustein, Phys. Rev. Lett. 58, 658 (1987).

2. J.D.Garrett and J.-Y.Zhang, Cocoyoc, 1988, Book of Abtsracts ; J.-Y. Zhang, R. F. Casten, and D. S. Brenner, Phys. Lett. B 227, 1 (1989)

3. R. B. Cakirli, D. S. Brenner, R. F. Casten, and E. A. Millman, Phys. Rev. Lett. 94, 092501 (2005); 95, 119903(E) (2005)

4. M. Stoitsov, R. B. Cakirli, R. F. Casten, W. Nazarewicz, and W. Satula, Phys.Rev.Lett. 98 , 132502 (2007).

5. L. Chen et al., Phys.Rev.Lett. 102, 122503 (2009)

6. K. Heyde et al., Phys. Lett. 155B, 303 (1985), P. Federman and S. Pittel, Phys. Lett. 69B, 385 (1977); Phys. Lett. B 77, 29 (1978), T. Motobayashi et al., Phys. Lett. B 346, 9 (1995), R. V. F. 
Janssens et al., Phys. Lett. B 546, 55 (2002), J. Dobaczewski et al., Phys. Rev. Lett. 60, 2254 (1988), M. V. Stoitsov, J. Dobaczewski, W. Nazarewicz, and P. Borycki, Int. J. Mass Spectrom. 251, 243 (2006)

7. D. Neidherr et al., Phys. Rev. C 80, 044323 (2009).

8. P. Van Isacker, D.D. Warner and D.S. Brenner, Phys. Rev. Lett. 74, 4607 (1995).

9. D.S. Brenner, C. Wesselborg, R.F. Casten, D.D. Warner and J.-Y. Zhang, Phys. Lett. 243 B, 1 (1990).

10. E. P. Wigner, Phys. Rev. 51, 106 (1937).

11. R.B. Cakirli, K. Blaum, R.F. Casten, Phys. Rev. C 82, 061304 (R) (2010)

12. D. Bonatsos, S. Karampagia, R.B. Cakirli, R.F. Casten, K. Blaum, L. Amon Susam, in preparation

13. D. Bonatsos (private communication)

14. D. Shubina et al. (Phy. Rev. C, in press)

15. F. Iachello and A. Arima, The Interacting Boson Model (Cambridge University Press, Cambridge, 1987).

16. R.B. Cakirli, R.F. Casten, R. Winkler, K. Blaum and M. Kowalska, Phys. Rev. Lett. 102, 082501 (2009). 